\title{
Impacto do déficit visual na qualidade de vida em idosos usuários do sistema único de saúde vivendo no sertão de Pernambuco
}

\author{
Visual impairment impact on the quality of life of the elderly population that uses the public health care \\ system from the western countryside of Pernambuco State, Brazil
}

Vasco Torres Fernandes Bravo Filho ${ }^{1}$, Roberta Urbano Ventura ${ }^{2}$, Carlos Teixeira Brandt ${ }^{3}$, Camila Sarteschi ${ }^{4}$, Marcelo Carvalho Ventura $^{1}$

\section{RESUMO}

Objetivo: Avaliar o impacto das doenças oculares sobre a qualidade de vida de uma população idosa do sertão de Pernambuco, localizado na região nordeste do Brasil. Métodos: Foram entrevistados 580 indivíduos acima de 59 anos, por meio do questionário de avaliação de qualidade de vida "Visual Functioning Questionnaire" (VFQ). Todos os indivíduos foram submetidos a exame oftalmológico completo. Os resultados das variáveis quantitativas foram expressos por suas médias e desviospadrão. Os resultados das variáveis qualitativas foram expressos por suas frequências absolutas e relativas.

Resultados: A média de idade foi de $70 \pm 8,1$ anos. Cerca de $86,0 \%$ dos entrevistados declararam ser analfabetos ou ter o ensino fundamental incompleto. As principais queixas foram: baixa visual $(71,1 \%)$ e ardor/prurido $(69,0 \%)$. A acuidade visual não era normal em 37,4\% dos idosos. Por volta de 75,0\% dos entrevistados relataram ter saúde regular ou ruim, e 77,0\% diziam ter uma visão regular ou ruim. A qualidade de vida foi considerada pior conforme a piora da condição visual do idoso.

Conclusão: $\bigcirc$ déficit visual representou um impacto negativo sobre a qualidade de vida dos idosos do sertão Pernambucano.

Descritores: Idoso; Qualidade de vida; Acuidade visual; Brasil

\begin{abstract}
Purpose: To evaluate the impact of visual impairment on the quality of life of the elderly population living in the western countryside of Pernambuco State, located in northeast of Brazil.

Methods: Five hundred and eighty subjects over 59 years of age were interviewed using the Visual Functioning Questionnaire (VFQ). All subjects underwent complete eye examination. The results of the quantitative parameters were expressed by mean $\pm S D$. The results of categorical parameters were expressed by their frequencies.

Results: Mean age was of $70 \pm 8.1$ years. About $86 \%$ of the interviewed elderly were illiterate or had incomplete education. The mean complaints were low visual acuity (71.1\%) and itching/burning (69.0\%). The visual acuity was not normal in $37.4 \%$ of the elderly. About $75.0 \%$ of the subjects reported to have regular or bad health, and $77.0 \%$ reported to have regular or bad vision. The reported quality of life decreased according to the worsening of the visual acuity.
\end{abstract}

Conclusion: Visual impairment had a negative impact on the quality of life of the elderly population from the western countryside of Pernambuco State.

Keywords: Aged; Quality of life; Visual acuity; Brazil

\section{INTRODUÇÃO}

O processo de envelhecimento da população brasileira vem sendo enfatizado(1). No último censo do IBGE, no ano 2010, o porcentual de pessoas idosas, no Brasil, era cerca de 10,8\% do total da população, correspondendo a aproximadamente 20 milhões de pessoas com 60 anos ou mais. No Estado de Pernambuco esse porcentual era por volta de $10,7 \%$ da população(2).

O envelhecimento da população traz como consequência o aumento da prevalência das doenças crônicas, características do idoso ${ }^{(3)}$, dentre estas, encontram-se algumas doenças oculares. Ao longo dos dos anos, as estruturas oculares sofrem de uma forma cumulativa os inúmeros danos metabólicos e ambientais. Com isso, as formas mais comuns de doenças oculares são mais frequentes e mais debilitantes nos idosos ${ }^{(4)}$
Outros fatores importantes relacionados à perda visual são; sexo, geografia, alimentação e o clima (exposição ao sol). Fatores sociais e econômicos também têm influência na saúde ocular do idoso(5).

Existem aproximadamente 135 milhões de deficientes visuais no mundo, sendo que 45 milhões são classificados como cegas (acuidade visual pior ou igual a 20/200 no melhor olho)(6).

O impacto da perda da capacidade visual acarreta consequências adversas em nível individual e coletivo, dando origem a problemas psicológicos, sociais, econômicos, pois implica em perda de autoestima, de status, em restrições ocupacionais e em consequente diminuição de renda. Para a sociedade, representa um importante encargo oneroso e perda de força de trabalho. O déficit visual leva a diminuição na qualidade de vida dos idosos ${ }^{(7,8)}$, estando associado à maiores taxas de suicídio nessa população( ${ }^{(9)}$.
Submetido para publicação: 22 de agosto de 2011

Aceito para publicação: 21 de março de 2012

Trabalho realizado no Departamento de Saúde Ocular da Fundação Altino Ventura - FAV - Recife (PE), Brasil.

${ }^{1}$ Médico, Curso de Especialização em Oftalmologia da Fundação Altino Ventura - FAV - Recife (PE), Brasil.

Médica, Departamento de Córnea e Doenças Externas Oculares e Cirurgia Refrativa, Fundação Altino Ventura - FAV - e do Hospital de Olhos de Pernambuco - HOPE - Recife (PE), Brasil.

3 Professor, Departamento de Cirurgia Pediátrica, Universidade Federal de Pernambuco - UFPE Recife (PE), Brasil.

${ }^{4}$ Estatística, pela Escola de Saúde Publica, Universidade de São Paulo - USP - São Paulo (SP), Brasil.
Financiamento: Não houve financiamento para este trabalho.

Divulgação de potenciais conflitos de interesse: V.T.F.Bravo Filho, Não; R.U.Ventura, Não; C.T.Brandt, Não; C.Sarteschi, Não; M.C.Ventura, Não.

Endereço para correspondência: Fundação Altino Ventura - FAV. Vasco Torres Fernandes Bravo Filho Rua da Soledade, 170 - Recife (PE) - 50070-040 - Brasil - E-mail: vascobravofilho@yahoo.com.br 
É fundamental detectar a condição visual da população idosa, avaliando-se o quanto sua qualidade de vida está prejudicada em virtude do déficit visual. Estes dados possibilitarão intervir nas doenças oculares passíveis de tratamento tais como catarata e ametropias, visando melhorar a qualidade de vida e a saúde ocular dos idosos ${ }^{(7,8)}$.

Os instrumentos de qualidade de vida são úteis na avaliação do custo/benefício do tratamento para doenças dos idosos ${ }^{(10)}$. A maioria destes instrumentos é aplicável apenas àquela cultura na qual foram desenvolvidos e testados ${ }^{(11)}$.

Este trabalho tem como propósito avaliar a condição de saúde ocular e o impacto do déficit visual na qualidade de vida em idosos do sertão de Pernambuco.

\section{MÉTODOS}

Trata-se de estudo observacional de corte transversal, com base populacional, sobre o impacto das doenças oculares dos idosos e das queixas visuais na qualidade de vida.

A população alvo foi composta por indivíduos, usuários do sistema único de saúde, de ambos os sexos, residentes nos municípios de Salgueiro e Ibimirim, sertão da região nordeste do Brasil, e que atenderam aos seguintes critérios: idade igual ou superior a 60 anos; aceitar participar livremente do estudo após o esclarecimento e assinatura do termo de consentimento livre e esclarecido.

Os dados foram coletados na unidade oftalmológica móvel da Fundação Altino Ventura por meio de entrevistas estruturadas, contendo informações de identificação, sociodemográficos, dados clínicos gerais e oftalmológicos, qualidade de vida e exame oftalmológico completo a fim de detectar o impacto na qualidade de vida das principais doenças oculares que acometem essa população. As entrevistas foram realizadas por oftalmologistas, alunos do curso de especialização em oftalmologia, pediatra e assistentes sociais devidamente capacitados.

As variáveis de interesse foram as seguintes: variáveis sociodemográficas [sexo, idade, raça, estado civil, escolaridade, profissão, renda familiar (SM), tipo de moradia]; variáveis clínicas gerais [comorbidades (HAS, DM, etc.), alergia, distúrbios da tireoide, doença reumatológica, pressão arterial, peso/altura, atividade física, tabagismo, consumo de álcool, uso de medicação crônica]; variáveis clínicas oftalmológicas (alteração na acuidade visual, sintomas oculares, gravidade, antecedente familiar, cirurgia ocular prévia, conjuntivite, utilização de óculos, conhecimento da doença); variáveis do exame oftalmológico (acuidade visual, anamnese, ectoscopia, exame refracional, exame de biomicroscopia, tonometria de aplanação, fundoscopia direta e indireta); variáveis de qualidade de vida [National Eye Institute Visual Functioning Questionnaire - 25 (VFQ-25) adaptado].

Antes do exame oftalmológico completo, todos os pacientes foram submetidos ao questionário, onde foram informados os dados referentes às variáveis de interesse.

De acordo com Organização Mundial de Saúde (OMS), a visão é classificada em normal (acuidade visual no melhor olho melhor ou igual a 20/60), subnormal (acuidade visual entre 20/70 a 20/150 no melhor olho) e cegueira (acuidade visual pior ou igual a 20/200 no melhor olho).

O tamanho amostral foi tomado considerando as frequências das doenças oculares mais prevalentes (catarata, glaucoma, doença macular relacionada à idade e retinopatia diabética) em idosos, sendo considerado o número mínimo de 400 indivíduos em Salgueiro e de 140 em Ibimirim.

Os resultados foram apresentados sob a forma de tabelas. As variáveis qualitativas foram ilustradas em frequências absolutas (N) e relativas (porcentual). Para as variáveis quantitativas foram utilizadas médias e medianas para resumir as informações, e desvios-padrão, mínimo e máximo para indicar a variabilidade dos dados. Para a análise comparativa e inferencial foram utilizados testes estatísticos adequados para cada análise desejada. Foi usado $p<0,05$ para rejeição da hipótese de nulidade.
Os pacientes foram informados, em linguagem acessível, que estavam participando de pesquisa clínica e assinaram o termo de consentimento livre e esclarecido.

O projeto foi aprovado pelo Comitê de Ética em Pesquisa em Seres Humanos da Fundação Altino Ventura (FAV).

\section{RESULTADOS}

Foram examinados 580 indivíduos com idade igual ou superior a 60 anos, residentes nos municípios de Salgueiro e lbimirim e usuários do sistema único de saúde.

A idade variou de 60 a 98 anos, com média de $70 \pm 8,1$ anos, sendo $83,6 \%$ dos indivíduos com idade entre 60 e 79 anos. A maioria dos idosos foi do sexo feminino $(59,1 \%)$. Eram analfabetos ou tinham o ensino fundamental incompleto $86,2 \%$ dos entrevistados. A renda familiar média foi de 1,5 salários mínimo (cerca de 440 dólares americanos), entretanto, $60,6 \%$ dos idosos tinham renda familiar inferior ou igual a um salário mínimo. Cerca de $67,0 \%$ dos idosos moravam em zona rural.

Ao se questionar os indivíduos de acordo com os sintomas oculares, as principais queixas foram baixa visual $(71,1 \%)$ e ardor/prurido (69,0\%).

Mais de $60 \%$ dos indivíduos apresentavam sobrepeso ou algum grau de obesidade e eram tabagistas, e 69,1\% estavam com a pressão arterial alterada (PAS $\geq 140$ ou PAD $\geq 90 \mathrm{mmHg}$ ) no momento do exame.

Entre as comorbidades, a hipertensão arterial correspondeu a $70,6 \%$ em seguida da diabetes com $14 \%$.

Cerca de $63,0 \%$ dos idosos apresentavam ao exame acuidade visual normal sem correção. A acuidade visual não foi normal em $37,4 \%$ dos idosos (Tabela 1). Ao se questionar os antecedentes, por volta de $25 \%$ dos pacientes relataram história de cegueira na família.

Entre os pacientes, cerca de 18\% fazia o primeiro exame oftalmológico. Daqueles que já haviam feito alguma consulta antes, aproximadamente $73 \%$ realizaram o exame há mais de um ano. Cerca de 23\% dos examinados já tinham sido submetidos a alguma cirurgia ocular.

A catarata foi a doença ocular mais prevalente, presente em 45,0\% dos idosos, seguida do glaucoma (18,1\%).

A primeira parte do questionário de qualidade de vida revelou que aproximadamente $75,0 \%$ dos entrevistados apresentavam saúde regular ou ruim, e 77,0\% referiram apresentar uma visão regular ou ruim.

A média do escore geral da qualidade de vida dos entrevistados foi de 76,2 $\pm 17,3$ (Tabela 2)

$\mathrm{Na}$ análise da qualidade de vida com relação à condição visual (normal X subnormal/cegueira) dos pacientes, foi aplicado o teste não paramétrico de Mann-Whitney. A análise foi feita para o escore total de qualidade de vida e também para os subdomínios do questionário de qualidade de vida. Verificou-se que a qualidade de vida diminuiu conforme a piora da condição visual do idoso (Tabela 2).

Para a comparação dos escores de qualidade de vida em relação à idade foi aplicado o teste não paramétrico de Mann-Whitney. Observou-se diferença estatisticamente significante do escore de

\begin{tabular}{|c|c|c|}
\hline Condição visual & $\mathbf{N}$ & $\%$ \\
\hline Normal & 360 & 62,6 \\
\hline Subnormal & 135 & 23,5 \\
\hline Cegueira & 80 & 13,9 \\
\hline Total & 575 & 100,0 \\
\hline
\end{tabular}


Tabela 2. Comparação da qualidade de vida segundo a condição visual dos idosos do Sertão de Pernambuco, utilizando-se o questionário Visual Functioning Questionnaire - $\mathbf{2 5}$ adaptado e a classificação de visão da OMS (normal: acuidade visual melhor ou igual a 20/60 no melhor olho; subnormal: acuidade visual entre $20 / 70$ a 20/150 no melhor olho; cegueira: acuidade visual pior ou igual a 20/200 no melhor olho)

\begin{tabular}{|c|c|c|c|c|c|c|c|c|}
\hline Qualidade de vida & Visão & $\mathbf{N}$ & Média & Mediana & Desvio padrão & Mínimo & Máximo & p-valor \\
\hline \multirow[t]{3}{*}{ Qualidade de vida geral } & Normal & 360 & 79,3 & 84,5 & 14,6 & 12,5 & 97,8 & \\
\hline & Subnormal/Cegueira & 214 & 71,3 & 77,2 & 19,7 & 7,9 & 100,0 & $<0,001^{*}$ \\
\hline & Total & 574 & 76,3 & 81,5 & 17,1 & 7,9 & 100,0 & \\
\hline \multirow[t]{3}{*}{ Saúde geral } & Normal & 360 & 28,1 & 25,0 & 20,3 & 0 & 100,0 & \\
\hline & Subnormal/Cegueira & 214 & 25,3 & 25,0 & 19,4 & 0 & 100,0 & 0,122 \\
\hline & Total & 574 & 27,1 & 25,0 & 20,0 & 0 & 100,0 & \\
\hline \multirow[t]{3}{*}{ Visão } & Normal & 360 & 46,2 & 50,0 & 19,5 & 0 & 100,0 & \\
\hline & Subnormal/Cegueira & 214 & 40,3 & 50,0 & 22,0 & 0 & 100,0 & $<0,001^{*}$ \\
\hline & Total & 574 & 44,0 & 50,0 & 20,6 & 0 & 100,0 & \\
\hline \multirow[t]{3}{*}{ Dor ocular } & Normal & 360 & 74,1 & 75,0 & 21,7 & 12,5 & 100,0 & \\
\hline & Subnormal/Cegueira & 214 & 70,3 & 75,0 & 23,5 & 0 & 100,0 & 0,063 \\
\hline & Total & 574 & 72,7 & 75,0 & 22,5 & 0 & 100,0 & \\
\hline \multirow[t]{3}{*}{ Atividades para perto } & Normal & 359 & 79,2 & 83,3 & 22,3 & 0 & 100,0 & \\
\hline & Subnormal/Cegueira & 212 & 71,5 & 75,0 & 25,6 & 0 & 100,0 & $<0,001^{*}$ \\
\hline & Total & 571 & 76,3 & 83,3 & 23,8 & 0 & 100,0 & \\
\hline \multirow[t]{3}{*}{ Atividades para longe } & Normal & 359 & 80,1 & 87,5 & 21,6 & 8,3 & 100,0 & \\
\hline & Subnormal/Cegueira & 212 & 69,7 & 75,0 & 26,5 & 0 & 100,0 & $<0,001^{*}$ \\
\hline & Total & 571 & 76,7 & 83,3 & 24,0 & 0 & 100,0 & \\
\hline \multirow[t]{3}{*}{ Aspectos sociais } & Normal & 357 & 95,8 & 100,0 & 13,8 & 12,5 & 100,0 & \\
\hline & Subnormal/Cegueira & 209 & 87,4 & 100,0 & 22,2 & 12,5 & 100,0 & $<0,001^{*}$ \\
\hline & Total & 566 & 92,7 & 100,0 & 17,8 & 12,5 & 100,0 & \\
\hline \multirow[t]{3}{*}{ Saúde mental } & Normal & 360 & 79,9 & 87,5 & 20,3 & 0 & 100,0 & \\
\hline & Subnormal/Cegueira & 214 & 72,8 & 81,2 & 24,8 & 0 & 100,0 & $0,001^{*}$ \\
\hline & Total & 574 & 77,3 & 81,2 & 22,3 & 0 & 100,0 & \\
\hline \multirow[t]{3}{*}{ Atividades da vida diária } & Normal & 359 & 83,2 & 100,0 & 25,0 & 0 & 100,0 & \\
\hline & Subnormal/Cegueira & 212 & 75,2 & 87,5 & 28,6 & 0 & 100,0 & $<0,001^{*}$ \\
\hline & Total & 571 & 80,2 & 100,0 & 26,7 & 0 & 100,0 & \\
\hline \multirow[t]{3}{*}{ Dependência } & Normal & 359 & 90,9 & 100,0 & 18,6 & 0 & 100,0 & \\
\hline & Subnormal/Cegueira & 212 & 79,4 & 100,0 & 30,5 & 0 & 100,0 & $<0,001^{*}$ \\
\hline & Total & 571 & 86,6 & 100,0 & 24,3 & 0 & 100,0 & \\
\hline \multirow[t]{3}{*}{ Capacidade dirigir automóveis } & Normal & 35 & 81,1 & 87,5 & 22,6 & 12,5 & 100,0 & \\
\hline & Subnormal/Cegueira & 15 & 73,3 & 87,5 & 28,7 & 12,5 & 100,0 & 0,403 \\
\hline & Total & 50 & 78,7 & 87,5 & 24,5 & 12,5 & 100,0 & \\
\hline \multirow[t]{3}{*}{ Visão de cores } & Normal & 359 & 92,5 & 100,0 & 17,6 & 0 & 100,0 & \\
\hline & Subnormal/Cegueira & 208 & 84,7 & 100,0 & 25,6 & 0 & 100,0 & $<0,001^{*}$ \\
\hline & Total & 567 & 89,6 & 100,0 & 21,2 & 0 & 100,0 & \\
\hline \multirow[t]{3}{*}{ Visão periférica } & Normal & 358 & 86,9 & 100,0 & 21,9 & 0 & 100,0 & \\
\hline & Subnormal/Cegueira & 208 & 78,5 & 100,0 & 27,3 & 0 & 100,0 & $<0,001^{*}$ \\
\hline & Total & 566 & 83,8 & 100,0 & 24,3 & 0 & 100,0 & \\
\hline
\end{tabular}

* = estatisticamente significante

qualidade de vida geral e dos domínios visão, atividades de perto, atividades de longe, aspectos sociais, saúde mental, atividade da vida diária, dependência, visão de cores e periférica, entre os idosos com menos de 80 anos quando comparados com aqueles com 80 anos ou mais. Em todos esses casos, verifica-se que a qualidade de vida é melhor quanto menor a idade dos pacientes.
Ao analisar a escolaridade com os escores qualidade de vida foi utilizado o teste não paramétrico de Kruskall-Wallis. Observou-se que o escore de qualidade de vida geral e dos domínios saúde, atividades de perto, aspectos sociais, saúde mental, dependência, visão de cores e periférica, a qualidade de vida aumenta conforme o aumento da escolaridade. 
Para avaliar a relação do escores de qualidade de vida com o fato de o idoso trabalhar ou não foi aplicado o teste não paramétrico de Mann-Whitney. Os idosos que trabalhavam tinham maior qualidade de vida em relação àqueles que não exerciam nenhuma atividade profissional nos escores de qualidade de vida geral e nos domínios atividades de longe, aspectos sociais, saúde mental, atividades da vida diária, dependência e visão periférica.

A comparação dos escores de qualidade de vida com a renda familiar foi analisada por meio do teste não paramétrico de MannWhitney. Os idosos com renda familiar inferior ou igual a um salário mínimo tinham pior qualidade de vida em comparação com os idosos com renda maior que um salário mínimo apenas com relação aos escores de qualidade de vida geral.

\section{DISCUSSÃO}

Dentre os modelos de avaliação de qualidade de vida existentes, apenas o "Visual Function Questionnaire" (VFQ) e o "Activities of Daily Vision Scale" (ADVS) são utilizados para avaliar a qualidade de vida em doenças oculares ${ }^{(10)}$. O questionário "ADVS" apesar de ter 20 atividades relacionadas à visão, não aborda atividades do ponto de vista psicológico e do nível de dependência(12). Já o "VFQ" avalia tanto qualidade de vida como função visual ${ }^{(13)}$. Por tal motivo, a opção foi usar o "VFQ" como instrumento para avaliação da qualidade de vida dos idosos; além disso, este questionário já foi validado para utilização com a população brasileira ${ }^{(10)}$.

O déficit na acuidade visual representou impacto negativo sobre a qualidade de vida dos idosos da população estudada.

As dificuldades apresentadas pelos idosos não foram apenas relacionadas à leitura ou à atividade de perto, embora muitos relatassem analfabetismo e pouca atividade de leitura diária. O déficit visual trouxe comprometimentos em diversas atividades dos idosos. As atividades de perto dos idosos com visão subnormal (acuidade visual entre 20/70 a 20/150 no melhor olho) ou pior foram claramente afetadas, assim como as atividades de longe. Consequentemente, as atividades de vida diária foram prejudicadas nesses idosos com déficit visual. Todo esse impacto do déficit na visão acarreta uma maior dependência desses idosos. Tal dependência está intimamente ligada a uma pior saúde mental nos idosos com visão subnormal ou cegos, assim como o prejuízo da vida social, dos relacionamentos com amigos e familiares. A visão de cores foi claramente prejudicada nos indivíduos com maior déficit visual. Tal fato também está ligado com a maior dependência desses idosos, visto que algumas atividades da vida diária precisam da diferenciação das cores para serem executadas. O déficit visual influencia não só a visão para perto e longe como também a visão periférica, levando os idosos a terem maior insegurança ao se locomover ou executar tarefas. Todos esses achados demonstram o grande impacto que o déficit visual traz à vida do idoso, assim como suas consequências interagem entre si, causando um ciclo vicioso

Ao se demonstrar que idosos com acuidade visual subnormal ou cegos possuíam escore de qualidade de vida inferior aos que possuíam visão normal, constata-se a importância do sentido da visão sobre a vida das pessoas. Indivíduos sem déficit visual possuíam escore de saúde mental maior. Tal achado vem sugerir que o déficit visual pode estar associado com um maior risco de depressão(9). Os idosos com pior visão possuem maior mortalidade ${ }^{(9)}$. Todos esses achados ratificam a importância de se intervir em políticas públicas para diminuição do impacto do déficit visual sobre a vida dos indivíduos, pois além de trazer benefícios na qualidade de vida desta população, poderia haver menor dependência aos serviços previdenciários e diminuir a utilização dos serviços de saúde.

Mesmo acima dos 60 anos pode-se verificar diferença na qualidade de vida quando se compara indivíduos abaixo e acima dos 80 anos. Tal achado é fundamental, principalmente no momento atual onde há um envelhecimento da população mundial com conse- quente aumento na expectativa de vida(2), pois não há motivos para evitar intervenções em idosos para se melhorar a acuidade visual, desde que seja possível.

A escolaridade, como esperado, também mostrou impacto sobre a qualidade de vida dos idosos, sendo evidenciado que aqueles com maior grau de instrução possuíam melhor qualidade de vida. Isso vem demonstrar a importância de se estruturar as políticas públicas desde cedo, pois o gasto em educação na verdade se trata de investimento futuro e que trará grandes benefícios em todos os aspectos ao ser humano.

Assim como constatado em estudo australiano(5) o fato de o idoso continuar trabalhando demonstrou melhor qualidade de vida em comparação àqueles que não se mantiveram na população economicamente ativa. Isso demonstra que o fato do idoso se manter ativo mental e fisicamente melhoram seu desempenho nos afazeres diários, desenvolvendo melhor independência e autonomia ${ }^{(14)}$.

Obviamente, uma renda familiar menor estaria associada a uma menor qualidade de vida. Porém, o interessante é notar que mesmo um salário mínimo a mais traz impacto positivo sobre a qualidade de vida do idoso. Tal resultado vem ratificar a importância do idoso continuar em atividade econômica para melhorar sua renda, acarretando em duplo benefício à sua vida.

Até o momento, não foi realizado nenhum estudo da qualidade de vida nesta população do sertão pernambucano. Isso demonstra a importância deste trabalho para melhor entendimento desta população, que por morar na região nordeste do Brasil apresenta condições socioeconômicas desfavoráveis, visto que por muitos anos essa região não recebeu atenção das políticas públicas para beneficiar esta população. Mesmo com a melhora na condição de vida dos que moram nessa região ultimamente, áreas como o sertão continuam a ser carentes de recursos para investimento em saúde. Essa população do sertão em sua maioria possui baixa renda e o sistema único de saúde, que é direito de todo cidadão brasileiro de maneira gratuita, é a única opção de acesso a qualquer serviço de saúde. Apesar da ideia do sistema único de saúde ser excelente, na maioria das cidades, principalmente as de zona rural, esse acesso é difícil e muitas vezes as pessoas precisam se deslocar para grandes cidades para terem tratamento adequado e eficiente. Com o entendimento dessa problemática, os dados desse estudo poderão servir para orientação de novas políticas públicas de intervenção para melhorar a qualidade de vida dos idosos dessa região.

\section{CONCLUSÃO}

O déficit visual esteve associado a um impacto negativo sobre a qualidade de vida dos idosos vivendo no sertão de Pernambuco. Cerca de 37,0\% da população estudada apresentou déficit visual. O aumento na prevalência das doenças relacionadas à visão e sua consequente associação com a redução do bem-estar mostram a necessidade do aumento na prestação dos serviços de saúde oftalmológicos da população idosa, além de um maior suporte global ao idoso. Uma melhor acuidade visual e diversos fatores relacionados à vida diária estão relacionados com uma melhora na qualidade de vida desta população.

\section{REFERÊNCIAS}

1. Ramos LR, Veras RP, Kalache A. Envelhecimento populacional: uma realidade brasileira. Rev Saúde Pública. 1987;21(3):211-24.

2. Instituto Brasileiro de Geografia e Estatística - IBGE. Censo Demográfico de 2010. Disponível em: http://www.censo2010.ibge.gov.br/

3. Gottlie MGV, Carvalho D, Schneider RH, Cruz IBM. Aspectos genéticos do envelhecimento e doenças associadas: uma complexa rede de interações entre genes e ambiente. Rev Bras Geriatr Gerontol. 2007;10(3):273-83.

4. Keane EM, O'Connor M, Coakley D, Walsh JB. Eye screening in the elderly. Ir Med J. 1997; 90(4):141-2.

5. Taylor HR. Prevalence and causes of blindness in Australian aborigenes. Med J Aust. 1980;1(2):71-6. 
6. WHO Monitoring Committee for the Elimination of Avoidable Blindness. Vision 2020 The Right to Sight: the global initiative for the elimination of avoidable blindness. Report of the first meeting. Geneva, 17-18 January 2006. Geneva: World Health Organization; 2006.

7. Broman AT, Munoz B, Rodriguez J, Sanchez R, Quigley HA, Klein R, et al. The impact of visual impairment and eye disease on vision-related quality of life in a MexicanAmerican population: proyecto VER. Invest Ophthalmol Vis Sci. 2002;43(11):3393-8.

8. Chia EM, Wang JJ, Rochtchina E, Smith W, Cumming RR, Mitchell P. Impact of bilateral visual impairment on health-related quality of life: the Blue Mountains Eye Study. Invest Ophthalmol Vis Sci. 2004;45(1):71-6.

9. Tournier M, Moride Y, Ducruet T, Moshyk A, Rochon S. Depression and mortality in the visually-impaired, community-dwelling, elderly population of Quebec. Acta Ophthalmol. 2008;86(2):196-201.
10. Ferraz EVAP, Lima CA, Cella W, Arieta CEL. Adaptação de questionário de avaliação da qualidade de vida para aplicação em portadores de catarata. Arq Bras Oftalmol. 2002;65(3):293-8.

11. Kuyken W, Orley J, Hudelson P, Sartorius N. Quality of life assessment across cultures. Int J Ment Health.1994;23:5-27.

12. Mangione CM, Phillps RS, Seddon JM, Lawrence MG, Cook EF, Dailey R, et al. Development of the 'Activities of Daily Vision Scale'. A measure of visual functional status. Med Care. 1992:30(12):1111-26.

13. Mangione CM, Lee PP, Gutierrez PR, Spritzer K, Berry S, Hays RD; National Eye Institute Visual Function Questionnaire Field Test Investigators. Development of the 25-item National Eye Institute Visual Function Questionnaire. Arch Ophthalmol. 2001; 119(7):1050-8.

14. Amaral PN, Pomatti DM, Fortes VLF. Atividades físicas no envelhecimento humano: uma leitura sensível criativa. Rev Bras Ciênc Envelhec Hum. 2007;4(1):18-27.

\title{
XX Congresso Brasileiro de
} Prevenção da Cegueira e Reabilitação Visual

\author{
12 a 15 de setembro de 2012 \\ Parque Anhembi \\ São Paulo (SP)
}

Informações:

Tel.: (11) 5081-7028

Site: www.fernandapresteseventos.com.br

E-mail: cbo2012@fernandapresteseventos.com.br 